Santa Clara University

Scholar Commons

Electrical Engineering

School of Engineering

$12-20-2010$

\title{
Extraction of contact resistance in carbon nanofiber via interconnects with varying lengths
}

Ken Li

Patrick Wilhite

Santa Clara University, pwilhite@scu.edu

Vinit Khera

Shoba Krishnan

Santa Clara University, skrishnan@scu.edu

Xuhui Sun

See next page for additional authors

Follow this and additional works at: https://scholarcommons.scu.edu/elec

\section{Recommended Citation}

K. Li, R. Wu, P. Wilhite, V. Khera, S. Krishnan, X. Sun, and C.Y. Yang, "Extraction of contact resistance in carbon nanofiber via interconnects with varying lengths," Applied Physics Letters 97, 253109 (3 pp) (2010). https://doi.org/10.1063/1.3527927

Copyright (C) 2010 American Institute of Physics Publishing. Reprinted with permission.

This Article is brought to you for free and open access by the School of Engineering at Scholar Commons. It has been accepted for inclusion in Electrical Engineering by an authorized administrator of Scholar Commons. For more information, please contact rscroggin@scu.edu. 
Authors

Ken Li, Patrick Wilhite, Vinit Khera, Shoba Krishnan, Xuhui Sun, and Cary Y. Yang 


\title{
Extraction of contact resistance in carbon nanofiber via interconnects with varying lengths
}

\author{
$\mathrm{Ke} \mathrm{Li},{ }^{1}$ Raymond Wu, ${ }^{1}$ Patrick Wilhite, ${ }^{1}$ Vinit Khera, ${ }^{1}$ Shoba Krishnan, ${ }^{1}$ Xuhui Sun, ${ }^{2, a)}$ and \\ Cary Y. Yang ${ }^{1}$ \\ ${ }^{1}$ Center for Nanostructures, Santa Clara University, Santa Clara, California 95053, USA \\ ${ }^{2}$ Institute of Functional Nano and Soft Materials (FUNSOM), Soochow University, Suzhou 215123, \\ People's Republic of China
}

(Received 10 November 2010; accepted 23 November 2010; published online 20 December 2010)

\begin{abstract}
A method to extract the contact resistance and bulk resistivity of vertically grown carbon nanofibers $(\mathrm{CNFs})$ or similar one-dimensional nanostructures is described. Using a silicon-compatible process to fabricate a terrace test structure needed for the CNF length variation, the contact resistance is extracted by measuring in situ the resistances of individual CNFs with different lengths and within a small range of diameters using a nanoprober inside a scanning electron microscope. Accurate determination of contact resistances for various combinations of catalysts and underlayer metals can lead to eventual optimization of materials' growth and device fabrication processes for CNF via interconnects. ㅇ 2010 American Institute of Physics. [doi:10.1063/1.3527927]
\end{abstract}

Copper interconnects used in current complementary metal-oxide-semiconductor (CMOS) technology are rapidly approaching its scaling limit because of increasing resistivity due to grain boundary scattering and surface scattering, as well as reliability problems arising from electromigration. One-dimensional carbon nanostructures such as carbon nanotubes (CNTs) and CNFs provide an alternative to copper, due to their high current-carrying capacity ${ }^{1-3}$ and their robust thermal and mechanical properties. ${ }^{4-7}$ To better assess the potential of these nanostructures for use in nextgeneration integrated circuits, their electrical properties need to be extensively characterized and well understood. One of the key properties in a vertical via interconnect is the contact resistance between the nanostructure and the metal underlayer. Minimizing contact resistance is paramount to the fabrication of performance-driven interconnects since contact resistance tends to dominate the overall resistance of the device in the nanoscale.

Standard two-point measurements are generally unable to isolate the contact resistance from the bulk resistance of the device under test. Combinations of two-point and fourpoint measurements have been used to extract contact resistance and bulk resistivity of horizontal nanostructures. ${ }^{8,9}$ While the electrical properties of CNT vias have been reported, ${ }^{10,11}$ it is nontrivial to perform four-point measurements on such vertical one-dimensional nanostructures. Thus a method to extract the contact resistance in a via structure is timely and critically needed.

Previously we reported contact resistance extraction for via structures from current-voltage measurements on single CNFs using conductive atomic force microscopy (C-AFM). ${ }^{12}$ By analyzing the dependence of the measured total resistance, as well as the contact resistance on CNF diameter, the metal-CNF contact resistance was extracted. The key assumptions of that method were that all measured devices had the same length, and that the diameter dependence of the contact resistance is predictable. In principle, a more straightforward approach would be to keep the diam-

\footnotetext{
${ }^{\text {a) }}$ Author to whom correspondence should be addressed. Electronic mail: xhsun@suda.edu.cn.
}

eter fixed and vary the length, as the contact resistance is clearly not a function of CNF length. In practice, however, it is difficult to control the CNF growth to yield a sufficiently large sample of test devices with the same or nearly the same diameter. Thus a certain diameter distribution within the sample becomes inevitable. Here we describe a method to extract contact resistance in a CNF via structure from the measured total resistance as a function of CNF length within a small range of diameters. A silicon-compatible test structure fabrication technique is used to yield the desired length variation while ensuring that all measured devices have undergone the same fabrication processes.

Small variations in CNF synthesis conditions can lead to very different properties of the resulting material. To ensure homogenous properties of all devices tested, all CNFs used in the measurements are from the same growth batch, and their lengths are altered postsynthesis. To achieve this, a terraced substrate is designed and fabricated by chemically etching a $\mathrm{Si}$ wafer in potassium hydroxide $(\mathrm{KOH})$. To ensure proper step formation, silicon nitride $\left(\mathrm{Si}_{3} \mathrm{~N}_{4}\right)$ is deposited on areas of the wafer as a mask, allowing only the area exposed to be etched. HF is then used to remove the nitride. This procedure results in the formation of one smooth step with sharp edges verified by three-dimensional profilometry. The process is then repeated several times allowing new areas of the substrate to be exposed along with the previously etched steps, ultimately resulting in the terrace structure, as shown schematically in Fig. 1. After the terraced structure is fabricated, the underlayer metal and the catalyst metal are deposited for CNF growth. Two different combinations of metal for CNF growth are chosen: a $20 \mathrm{~nm}$ Ni catalyst on an $80 \mathrm{~nm}$ $\mathrm{Ti}$ underlayer metal (denoted by $\mathrm{Ni} / \mathrm{Ti}$ ) and a $20 \mathrm{~nm} \mathrm{Ni}$ catalyst on an $80 \mathrm{~nm} \mathrm{Cr}(\mathrm{Ni} / \mathrm{Cr})$. Vertically aligned CNFs are then grown using dc plasma-enhanced chemical vapor deposition at $750{ }^{\circ} \mathrm{C} .{ }^{13}$ The entire wafer is then encapsulated in $\mathrm{SiO}_{2}$ formed by thermal decomposition of tetraethylorthosilicate (TEOS) at $380^{\circ} \mathrm{C}$. This provides structural rigidity as well as electrical isolation between CNFs. A chemicalmechanical planarization (CMP) process is carried out to create a flat surface and to expose the CNF tips. 40-nm-thick Pt electrodes are then deposited on a $5 \mathrm{~nm}$ Ti buffer layer for 


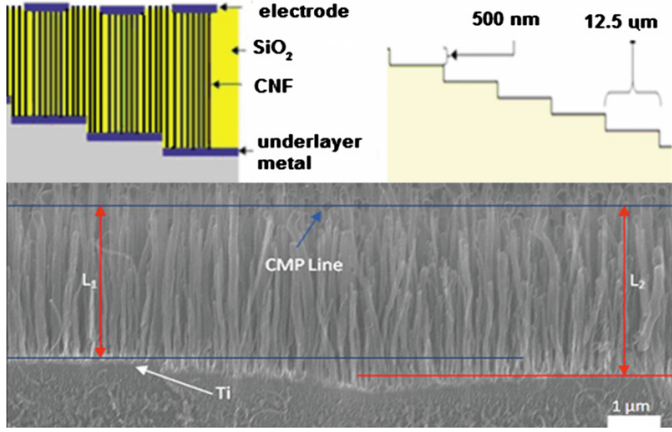

FIG. 1. (Color online) Top-right: schematic of partial terrace structure showing its dimensions used to create different lengths. Top-left: terrace structure after growth, TEOS, CMP, and metal pad deposition. Bottom: SEM image of two adjacent terrace steps with CNFs grown.

better adhesion. A scanning electron microscope (SEM) image of two adjacent terrace steps covered by CNFs is shown in Fig. 1.

$I-V$ measurements are performed in situ during SEM imaging with a nanoprober consisting of piezoactuated manipulators coupled to tungsten probes. One probe rests on a contact pad while the other lands on individual CNFs, as illustrated in Fig. 2. Initially, the $I-V$ curves for CNFs show non-Ohmic characteristics (nonlinearity), which are attributed to tunneling across the interface between each probe and the CNF. Current-stressing in the order of $10^{6} \mathrm{~A} / \mathrm{cm}^{2}$ is used to lessen the nonlinearity and to improve the contacts. This process reduces $R_{\text {total }}$ by up to an order of magnitude, but the result can lead to small variations in $R_{\text {total }}$ for CNFs with similar dimensions.

The measured resistance consists of

$$
R_{\mathrm{total}}=R_{\mathrm{pad}}+R_{\mathrm{CNF}}+R_{C}+R_{m}+R_{\text {probe-pad }}+R_{\text {probe-CNF}} .
$$

$R_{\text {pad }}$ includes the pad resistance in series with all CNFs under the pad and the contact resistance between them and the pad. $R_{m}$ is the resistance of the underlayer metal between the two probes. $R_{\text {probe-pad }}$ or $R_{\text {probe-CNF }}$ is the sum of probe resistances and contact resistances between the probes and pad or CNF, respectively. The sum of $R_{\text {pad }}, R_{m}$, and $R_{\text {probe-pad }}$ is estimated by measuring the resistance between any two pads on the same terrace step, given by

$$
R_{\text {pad-pad }}=2 R_{\text {pad }}+R_{m}+2 R_{\text {probe-pad }} \text {. }
$$

$R_{\text {total }}$ is generally in the $\mathrm{k} \Omega$ range, whereas the measured $R_{\text {pad-pad }}$ varies from a few $\Omega$ to $\sim 100 \Omega$. Therefore, $R_{\text {pad }}$, $R_{m}$, and $R_{\text {probe-pad }}$ are all negligible compared to $R_{\text {total }}$ and neglected in our analysis. The contact resistance between the tungsten probe tip and the CNF ( $\left.R_{\text {probe-CNF}}\right)$ is dependent on the pressure of the probe on the CNF. Even though this re-

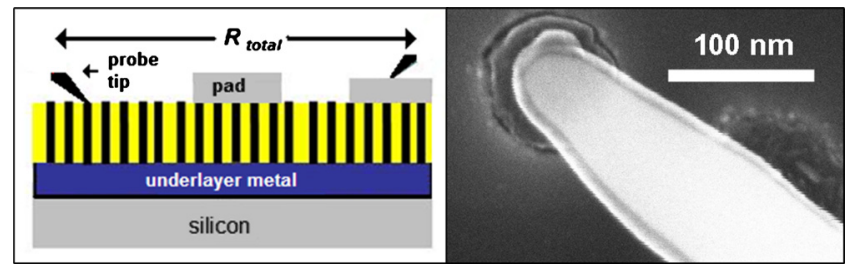

FIG. 2. (Color online) Left: schematic of one step of the terrace structure with probes on pad and exposed nanofibers. Right: SEM image of a landed probe tip on a single CNF.

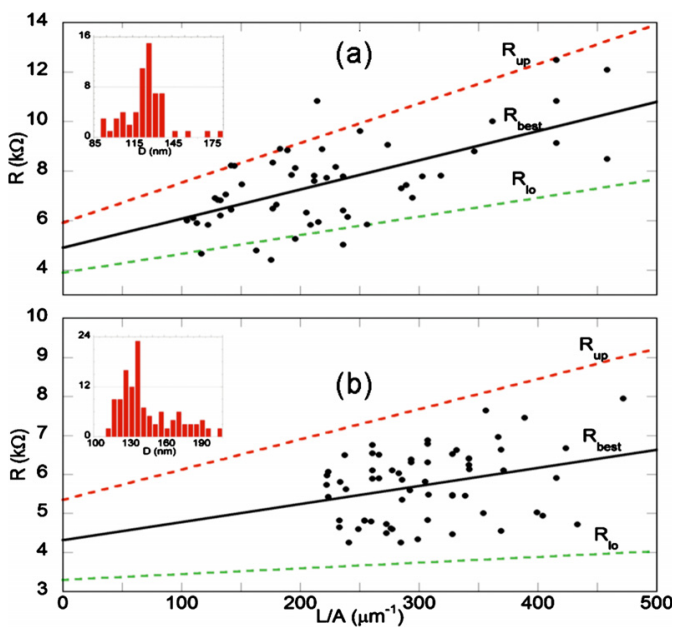

FIG. 3. (Color online) Measured resistance vs length results for (a) $\mathrm{Ni} / \mathrm{Ti}$ and (b) Ni/Cr. The lines are least-squares linear fit results for the best-fit (solid) and upper and lower limits (dashed), yielding contact resistance and resistivity. Insets are histograms showing the diameter distributions of the measured CNFs.

sistance can be minimized empirically, ${ }^{12}$ it is lumped into $R_{C}$ as the total contact resistance of the test device including the probe tip/CNF and the CNF/underlayer contacts. The measured resistance is then reduced to

$$
R_{\mathrm{total}} \approx R_{\mathrm{CNF}}+R_{C} .
$$

$I-V$ measurements are performed on CNFs on each terrace step. $R_{\text {total }}$ for each CNF is obtained as the reciprocal slope of the $I-V$ curve at $V=0$. The measured CNFs at the same step height are confined to a small area (less than 50 $\times 50 \mu \mathrm{m}^{2}$ ) due to variations that can result from CMP. After sufficient devices are measured to take into account statistical variations, the sample is then cleaved at the area where the measurements are taken and a SEM side-view image is obtained for determining the exact length. Although the fabrication of all devices used in this study was performed under the exact same conditions on the same wafer in order to minimize the inhomogeneity among individual CNFs, errors may still result from process variations and especially the diameter variation in device sampling. The method proposed here uses a statistical approach, and the more devices it includes, the more accurate it is.

For a Ni/Ti CNF sample, 61 devices were measured in different terraces corresponding to 6 different lengths; for $\mathrm{Ni} / \mathrm{Cr}, 121 \mathrm{CNFs}$ having 7 different lengths were measured. Based on the CNF diameter distributions shown in the insets of Fig. 3, a $45 \mathrm{~nm}$ window is chosen for our extraction scheme, which results in 100-145 $\mathrm{nm}$ for $\mathrm{Ni} / \mathrm{Ti}$ and $115-160$ $\mathrm{nm}$ for $\mathrm{Ni} / \mathrm{Cr} . R_{C}$ and $\rho$ are determined from the intercept and slope, respectively, of a least-squares linear fit to the measured $R_{\text {total }}$ versus $L / A_{\mathrm{CNF}}$, where $A_{\mathrm{CNF}}=\pi D_{\mathrm{CNF}}{ }^{2} / 4$ is the $\mathrm{CNF}$ cross-sectional area and $R_{\mathrm{total}}=\rho L / A_{\mathrm{CNF}}+R_{C}$ follows from Eq. (3). The data and results of the extraction are shown in Fig. 3. The extraction process is critically based on the assumption that $R_{C}$ is nearly constant over the chosen range of diameters. To test the validity of this assumption, we use our previously published model, ${ }^{12}$ as reproduced in Fig. 4 for Ni/Ti, to estimate the variation of $R_{C}$ in this diameter range and determine it to be about $0.5 \mathrm{k} \Omega$ for either sample. In addition, based on this model, $R_{C}$ varies as $1 / D_{\mathrm{CNF}}$, while $R_{\mathrm{CNF}} \propto 1 / D_{\mathrm{CNF}}{ }^{2}$, further supporting the con- 


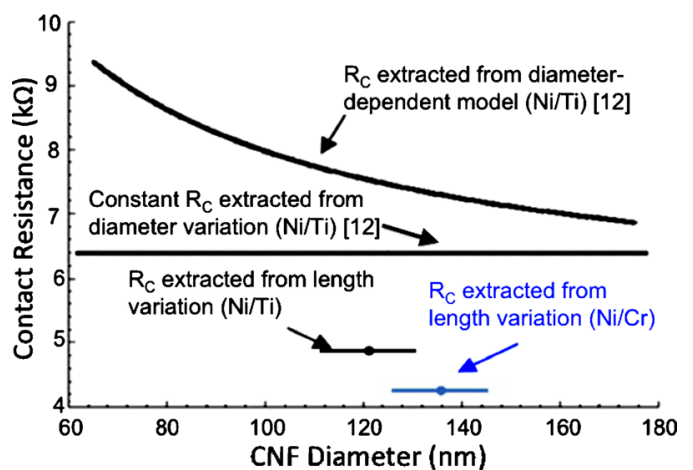

FIG. 4. (Color online) Comparison of various methods to extract contact resistance. The data represent the contact resistances extracted based on diameter variation, using constant- $R_{C}$ and diameter-dependent models (replotted from Ref. 12), and on length variation over a small range of diameters.

stant $R_{C}$ assumption. Therefore, for the measured resistance values of a few $\mathrm{k} \Omega$, such diameter variation has a little impact on our assumption of constant $R_{C}$ and the resulting extracted values.

To further examine our data and extraction scheme, in addition to obtaining the best linear fit (solid lines in Fig. 3), we compute the linear fits corresponding to the upper and lower limits for a two-standard-deviation variance of $L / A_{\mathrm{CNF}}$. These linear fits are indicated by the dashed lines in Fig. 3. This procedure provides us with a $95 \%$ confidence interval for results between the two limits, and it takes into account the somewhat random variations of measured total resistance due to current-stressing, as mentioned previously. The extracted parameters from the best-fit (best), upper (up), and lower $(l o)$ limit lines are given in Table I.

The best-fit extracted contact resistance of $\mathrm{Ni} / \mathrm{Cr}$ is found to be $4.3 \mathrm{k} \Omega$, while the CNF resistivity is 4.6 $\times 10^{-4} \Omega \mathrm{cm}$. The corresponding extracted contact resistance and CNF resistivity values for $\mathrm{Ni} / \mathrm{Ti}$ are $4.9 \mathrm{k} \Omega$ and $11.7 \times 10^{-4} \Omega \mathrm{cm}$, respectively. In comparison, our previous study using diameter variation yielded a contact resistance of $6.4 \mathrm{k} \Omega$ and a resistivity of $7.3 \times 10^{-4} \Omega \mathrm{cm}$ for similar CNFs on Ni/Ti devices. ${ }^{12}$ The resulting resistivity of the $\mathrm{CNFs}$ is comparable to that measured from horizontal CNFs. ${ }^{14,15}$ In general, contact resistance occurs due to formation of an electrical barrier between the CNF and the underlayer metal, due to the presence of an oxide or carbide interfacial layer or contaminants introduced during the synthesis and fabrication processes. It was shown that because of the relatively fast formation of $\mathrm{Al}_{2} \mathrm{O}_{3}$, using $\mathrm{Al}$ as an underlayer metal yielded a very large contact resistance. ${ }^{13}$ Here we find that for either underlayer metal, the CNF test device shows a much larger contact resistance than those for metal-metal contacts. X-ray photoelectron spectroscopy re-

TABLE I. Extracted values of $R_{C}$ and $\rho$ from the best, upper, and lower limit linear fits for CNFs grown with a $\mathrm{Ni}$ catalyst on $\mathrm{Cr}$ and $\mathrm{Ti}$ underlayers.

\begin{tabular}{lcc}
\hline \hline \multicolumn{1}{c}{ Sample } & $\mathrm{Ni} / \mathrm{Cr}$ & $\mathrm{Ni} / \mathrm{Ti}$ \\
\hline$R c_{\text {up }}(\mathrm{k} \Omega)$ & 5.1 & 5.9 \\
$R c_{\text {best }}(\mathrm{k} \Omega)$ & 4.3 & 4.9 \\
$R c_{\text {lo }}(\mathrm{k} \Omega)$ & 3.5 & 3.9 \\
$\rho_{\text {up }}(\Omega \mathrm{cm})$ & $7.0 \times 10^{-4}$ & $15.9 \times 10^{-4}$ \\
$\rho_{\text {best }}(\Omega \mathrm{cm})$ & $4.6 \times 10^{-4}$ & $11.7 \times 10^{-4}$ \\
$\rho_{\text {lo }}(\Omega \mathrm{cm})$ & $2.2 \times 10^{-4}$ & $7.5 \times 10^{-4}$ \\
\hline \hline
\end{tabular}

vealed that both the $\mathrm{Ti}$ and $\mathrm{Cr}$ underlayers had been oxidized after thermal annealing and $\mathrm{CNF}$ growth. ${ }^{13}$ The differences in contact resistance for CNFs grown on $\mathrm{Ni} / \mathrm{Ti}$ and $\mathrm{Ni} / \mathrm{Cr}$ are most likely due to dissimilar reaction kinetics in the early stages of the two growth processes, resulting in different interfaces between the CNF and the underlayer metal. In addition, the difference in the CNF resistivity between the $\mathrm{Ni} / \mathrm{Cr}$ and $\mathrm{Ni} / \mathrm{Ti}$ cases might be attributed to structural difference of CNFs grown on the two dissimilar underlayer metals, and partly due to the small contact resistance variation as a result of sampling CNFs with slightly different diameters. Nevertheless, in both cases, the contact resistance dominates the total resistance in the vertical CNF structure. Thus the terrace approach provides a platform to test various catalysts and underlayer metals, leading to the eventual optimization of the growth process and minimizing the contact resistance in via interconnects.

The similarities in results between the diameter variation ${ }^{12}$ and length-variation methods, as depicted in Fig. 4 , suggest that the diameter variation can be a convenient approach to extract contact resistance in vertical onedimensional nanostructures. However, because of the complex interface between the nanostructure and metal, which could result in equally complex transport mechanisms across it, the dependence of contact resistance on diameter is uncertain at best. ${ }^{12}$ Since contact resistance generally does not depend on the length of the nanostructure, the method of length-variation extraction should be considered more accurate and can be applied to any vertically aligned onedimensional nanostructures.

This work was supported by the United States Army Space and Missile Defense Command (SMDC) and carries Distribution Statement A, approved for public release, distribution unlimited.

${ }^{1}$ Z. Yao, C. L. Kane, and C. Dekker, Phys. Rev. Lett. 84, 2941 (2000).

${ }^{2}$ B. Q. Wei, R. Vajtai, and P. M. Ajayan, Appl. Phys. Lett. 79, 1172 (2001).

${ }^{3}$ A. Javey, P. Qi, Q. Wang, and H. Dai, Proc. Natl. Acad. Sci. U.S.A. 101, 13408 (2004).

${ }^{4}$ Q. Ngo, T. Yamada, M. Suzuki, Y. Ominami, A. M. Cassell, J. Li, M. Meyyappan, and C. Y. Yang, IEEE Trans. Nanotechnol. 6, 688 (2007).

${ }^{5}$ M. Suzuki, Y. Ominami, Q. Ngo, C. Y. Yang, A. M. Cassell, and J. Li, J. Appl. Phys. 101, 114307 (2007).

${ }^{6}$ M. A. Kuroda, A. Cangellaris, and J.-P. Leburton, Phys. Rev. Lett. 95, 266803 (2005).

${ }^{7}$ E. Pop, D. Mann, K. Goodson, and H. Dai, J. Appl. Phys. 101, 093710 (2007).

${ }^{8}$ A. Bachtold, M. Henny, C. Terrier, C. Strunk, C. Schonenberger, J. P. Salvetat, J. M. Bonard, and L. Forro, Appl. Phys. Lett. 73, 274 (1998),

${ }^{9}$ A. S. Walton, C. S. Allen, K. Critchley, M. L. Gorzny, J. E. McKendry, R. M. D. Brydson, B. J. Hickey, and S. D. Evans, Nanotechnology 18, 065204 (2007).

${ }^{10}$ A. Kawabata, S. Sato, T. Nozue, T. Hyakushima, M. Norimatsu, M. Mishima, T. Murakami, D. Kondo, K. Asano, M. Ohfuti, H. Kawarada, T. Sakai, M. Nihei, and Y. Awano, Proceedings of the IEEE 2008 International Interconnect Technical Conference, 2008, p. 237.

${ }^{11}$ J. C. Coiffic, M. Fayolle, H. Le Poche, S. Maitrejean, and S. Olivier, Proceedings of the IEEE 2008 International Interconnect Technical Conference, 2008, p. 153.

${ }^{12}$ W. Wu, S. Krishnan, T. Yamada, X. Sun, P. Wilhite, R. Wu, K. Li, and C. Y. Yang, Appl. Phys. Lett. 94, 163113 (2009).

${ }^{13}$ X. Sun, K. Li, R. Wu, P. Wilhite, T. Saito, J. Gao, and C. Y. Yang, Nanotechnology 21, 045201 (2010).

${ }^{14}$ T. Saito, T. Yamada, D. Fabris, H. Kitsuki, P. Wilhite, M. Suzuki, and C.Y. Yang, Appl. Phys. Lett. 93, 102108 (2008).

${ }^{15}$ T. Yamada, T. Saito, M. Suzuki, P. Wilhite, X. Sun, N. Akhavantafti, D. Fabris, and C. Y. Yang, J. Appl. Phys. 107, 044304 (2010). 